\title{
Supplementary Material: Wide-field multispectral super-resolution imaging using spin-dependent fluorescence in nanodiamonds
}

\author{
Edward H. Chen, Ophir Gaathon, Matthew E. Trusheim, \\ \& Dirk Englund*
}

\section{Supplementary Figures}

Autocorrelation of two NV site in bulk diamond In Fig. S1, we show the autocorrelation of the site shown in Fig. 2 of the main text. The anti-bunching dip of the normalized second order auto-correlation $\left(\mathrm{g}^{2}(\tau)\right.$ $=0.66$ ) therefore confirms the presence of no more than two emitters accounting for background.

Single $\mathrm{NV}^{-}$saturation curves on both an APD and emCCD In Fig. S2, we show the fluorescence of a single $\mathrm{NV}^{-}$centre saturating with respect to applied laser intensity using both an APD and the emCCD. The photons count rate collected on the emCCD was calculated using the calculations described in the Supplementary Methods below.

\section{Supplementary Methods}

Photon conversion calculation with emCCD

$$
G \times D U=\left(\gamma_{\text {signal }} \tau\right) Q E \times M+\left(\gamma_{D} \tau\right)
$$

where $\mathrm{G}$ is analog gain (electrons per ADU), DU is digital units on camera, $\gamma_{\text {signal }}$ is emission rate $(\mathrm{Hz}), \tau$ is camera exposure time (sec), $Q E$ is quantum efficiency in converting a photon to an electron, $M$ is the emCCD multiplication gain, and $\gamma_{D}$ is the dark count rate per pixel (Hz/pixel).

\section{Supplementary Equations and Discussion}

Derivation of spatial error as a function of laser power, exposure time, and number of emitters. When using a CCD detector, the fundamental localization error can vary significantly depending on the total magnification onto the camera. Light emitted from a point emitter and collected by a CCD detector will necessarily sacrifice spatial information due to the finite size of a pixel. If camera pixels did not have read-out

${ }^{*}$ Correspondence and requests for materials should be addressed to D. E. (englund@mit.edu) and E.H.C. (ehchen@mit.edu) 


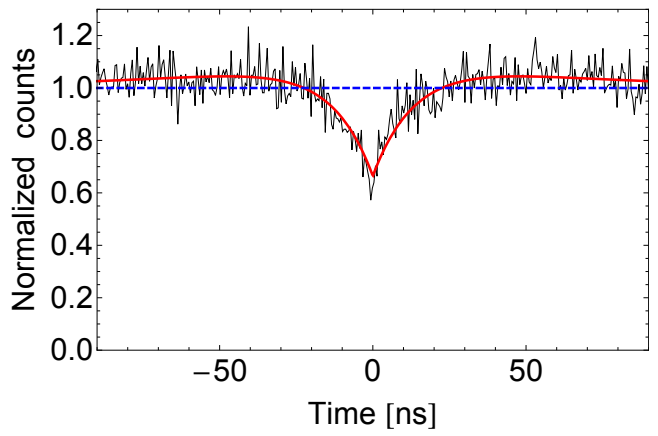

Figure S1: Normalized second order auto-correlation plot confirming the presence of no more than two emitters in the bulk sample shown in Fig. 2 of the main text.

a

b
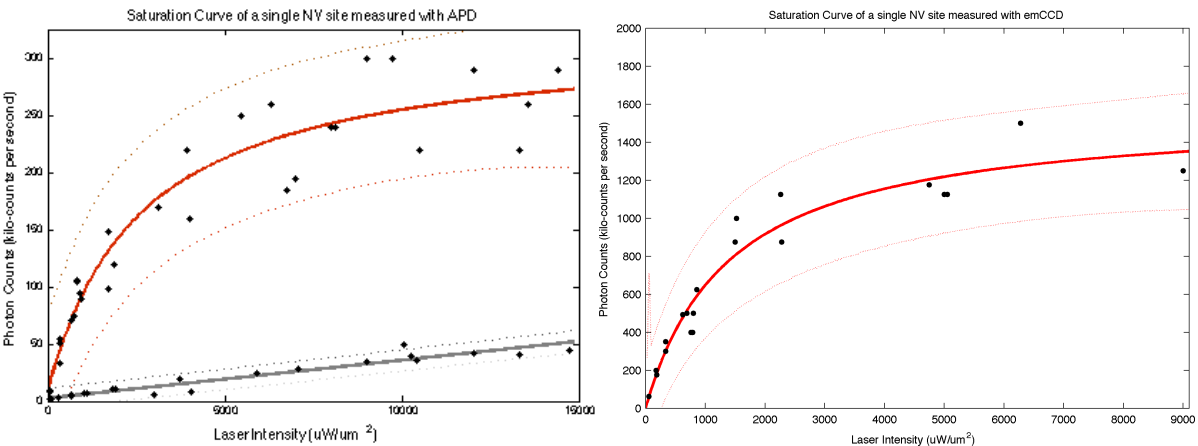

Figure S2: a. For the APD saturation curve, the red fit indicates a saturation count rate of 310 kilo-counts per second with a grey fit that shows background linearly increasing with incident laser intensity. b. A saturation curve taken with the CCD with a fitted curve indicating a saturation fluorescence rate of $\sim 1,600$ kilo-counts per second. 
noise or dark counts, then increasing magnification results in higher spatial resolution without any bound.

However, because pixels are of a finite size and have some noise, there exists an optimal magnification for optimal spatial localization (See Eqn. 1 [1]). Eqn. 1 below describes how magnification $(\sim s / a)$ and background noise $\left(\sigma_{\text {total }}\right)$ affect the standard error on the mean of a Gaussian function fit to the emission profile of a single emitter.

$$
\left\langle(\Delta \mathrm{x})^{2}\right\rangle=\frac{s^{2}+a^{2} / 12}{N}+\frac{8 \pi s^{4} \sigma_{t o t a l}^{2}}{a^{2} N^{2}}
$$

Because the demonstrated DESM technique relies on the $\mathrm{NV}^{-}$emitter, it is first important to describe its emission properties. The fluorescence rate from an $\mathrm{NV}^{-}$emitter depends on the intensity of the excitation laser, and can saturate at a maximum rate, $R_{\infty}$, past an excitation intensity limit, $I_{\text {sat }}$ (Eqn. 2a). In a DESM experiment, the signal, $N_{\text {photons }}$, is the lack of photons counts from a single $\mathrm{NV}^{-}$emitter when it is resonantly driven (Eqn. $2 \mathrm{~b}$ ), and the background noise, $\sigma_{\text {total }}$, of this emitter depends upon the number of other emitters within the same diffraction volume that are not being resonantly addressed, assuming a shot-noise limit (Eqn. 2c). The lack of collected photons is encapsulated by the contrast of the electron spin resonance measurement, $\mathrm{C}(\mathrm{I})$, which reaches a maximum of around $15-20 \%$ and is dependent on the incident laser power [2]. The number of photons collected by the CCD also depends on the system's total collection efficiency, $\eta$, and also the exposure time, $\tau$. The total background noise during a DESM measurement depends on the number of total emitters, $m$, in the diffraction volume since more emitters means the contrast compared with the background counts decreases. Finally, other contributions to background noise include background fluorescence that may increase with the pump laser, as parameterized by $\alpha$, and a constant background fluorescence, $\beta$.

$$
\begin{aligned}
C(I) & =\Theta \frac{\Omega_{r}^{2}}{\Omega_{r}^{2}+\Gamma_{p}^{\infty} \Gamma_{c}^{\infty}\left(\frac{s}{1+s}\right)^{2}}, \\
\Gamma_{\text {single }}(I) & =\frac{R_{\infty} I}{I_{\text {sat }}+I}, \\
N_{\text {photons }}(I, \tau) & =C(I) \eta \tau \Gamma_{\text {single }}(I) . \\
\sigma_{\text {total }}(m, I) & =\sqrt{\eta \tau \Gamma_{\text {single }}(I)((m-1)+(1-C(I)))+\alpha I+\beta} .
\end{aligned}
$$

Substitution of Eqn. 2 into Eqn. 1 and after a few steps of algebra gives 


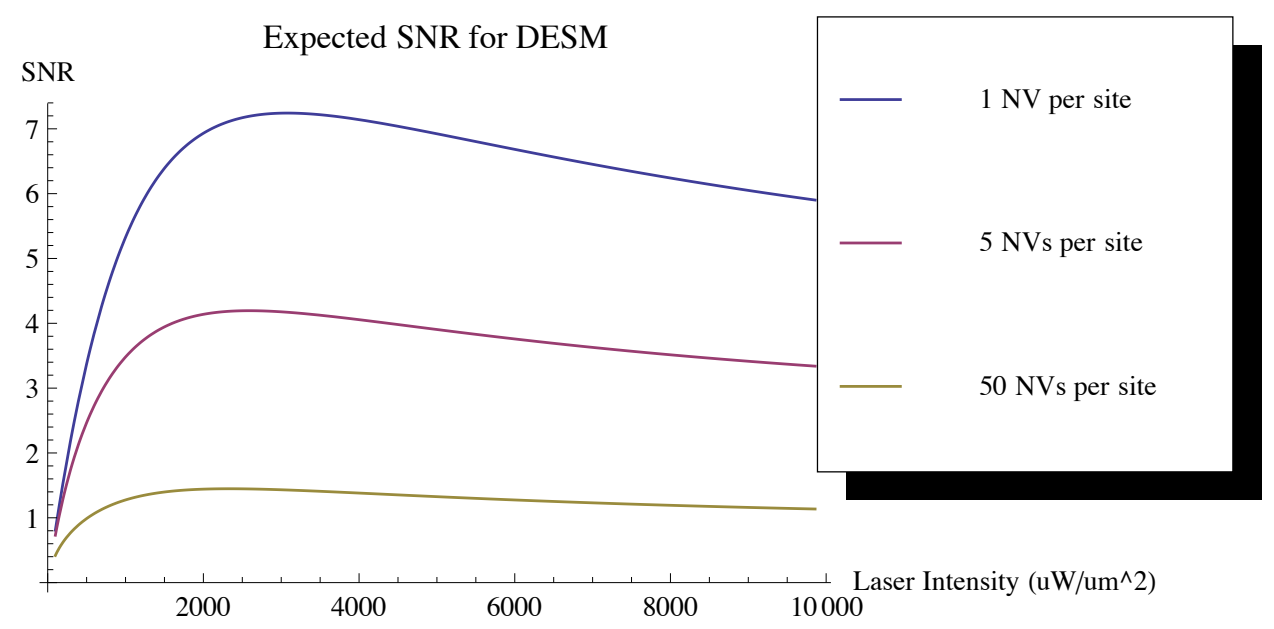

Figure S3: Theoretical signal-to-noise ratio with an emCCD. The signal is considered to be the contrast, $C(I)$, multiplied by the fluorescence from a single $\mathrm{NV}^{-}$collected at the shot-noise-limit.

the standard error in localizing the mean of a Gaussian function below:

$$
\begin{aligned}
\left\langle(\Delta \mathrm{x})^{2}\right\rangle & =\frac{s^{2}+a^{2} / 12}{N}+\frac{8 \pi s^{4} \sigma_{\text {total }}^{2}}{a^{2} N^{2}}, \\
& =\frac{s^{2}}{N_{\text {photons }}}\left\{1+\frac{1}{12}\left(\frac{a}{s}\right)^{2}+\frac{8 \pi}{\left(\frac{a}{s}\right)^{2}} \frac{\sigma_{\text {total }}^{2}}{N}\right\}, \\
& =\frac{s^{2}}{N_{\text {photons }}}\left\{1+\frac{1}{12}\left(\frac{a}{s}\right)^{2}+\frac{8 \pi}{\left(\frac{a}{s}\right)^{2}} \frac{\frac{N_{\text {photons }}}{C(I)}(m-1+1-C(I))+\alpha I+\beta}{N_{\text {photons }}}\right\}, \\
& =\frac{s^{2}}{N_{\text {photons }}}\left\{1+\frac{1}{12}\left(\frac{a}{s}\right)^{2}+\frac{8 \pi}{\left(\frac{a}{s}\right)^{2}} \frac{m-C(I)}{C(I)}\right\}+\mathcal{O}\left(\frac{1}{N_{\text {photons }}^{2}}\right) .
\end{aligned}
$$

In Fig. S3, we use the equations above to plot the signal-to-noise ratio as a function of laser intensity for $m=1,5$ and 50 emitters per diffraction limited spot. We estimate that for optimally resolving 5 emitters in a site up to $200 \mathrm{~kW} / \mathrm{cm}^{2}$ of laser intensity is required.

DESM in biological applications Nanodiamonds internalized by HeLa cells were shown to have little rotational diffusion in McGuinness 


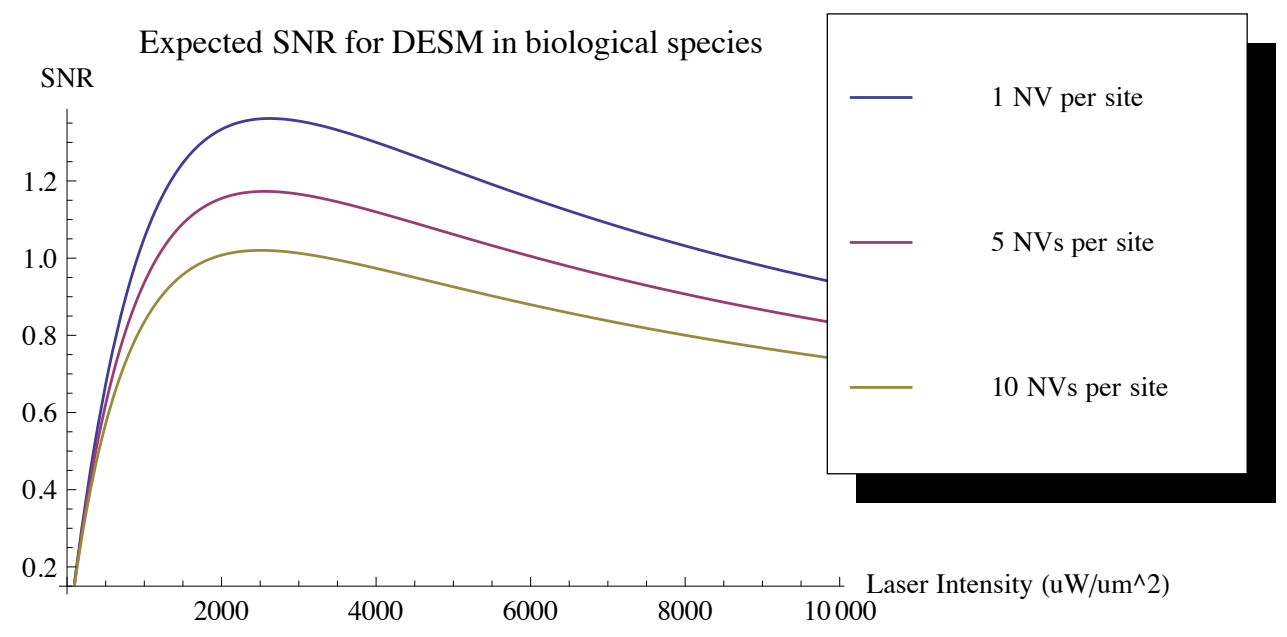

Figure S4: Estimated number of resolvable emitters given 5 times more background counts, and 5 times less collected photon counts from biologically ingested nanodiamonds.

et al. [3] For these $45 \mathrm{~nm}$-sized nanodiamonds, the angular rotation was estimated to be less than 10 degrees over several hours.

To account for the increased background and lower collection efficiency for imaging in biological samples, we refer to Equations 1 and 2 in the main text and Equation 3 in the supplementary. The fluorescence rate from single $\mathrm{NV}^{-}$centers was $\sim 100 \mathrm{kcps}$ compared with the maximal detected fluorescence detected from a stable NV in nanodiamond to be $\sim 500 \mathrm{kcps}$ in T. Schröder et al. [4] Assuming the collected fluorescence rate from biologically ingested nanodiamonds decreases by a factor of $5\left(R_{\infty} \rightarrow\right.$ $\left.R_{\infty} / 5\right)$ and the background counts increase by a factor of $5(\alpha \rightarrow \alpha \times 5)$, then the exposure time would need to increase by approximately a factor of $\sim 22$ to achieve the same SNR. In Figure S4, we estimate that in such a case DESM will still be able to address up to 10 unique centers per diffraction limited spot.

For freely rotating nanodiamonds, we expect a rate of $\sim 1 \mathrm{rad}^{2} / \mathrm{ms}$ for 50 $\mathrm{nm}$ nanodiamonds based on Maclaurin et al. [5] In such an environment, we expect that using a combination of solid immersion lens and a reflective top surface can increase the collection efficiency enough to collect 5-10x more fluorescence from a single NV. Thus based on our model, a unity SNR in the ODMR contrast can be achieved within $\sim 20$ us of exposure time, during which the nanodiamond is expected to rotate by 8 degrees. With such angular resolution, up to $\sim 10 \mathrm{NV}^{-}$orientations would be uniquely 
addressable (See Figure S5).

\section{Author Contributions}

E.H.C. and O.G. designed the experimental setup. E.H.C. performed the experiments and analysed the data; O.G. purified and prepared nanodiamonds; M.E.T. prepared samples and assisted in experimental setup; D.E. conceived the idea and supervised the project.

\section{References}

[1] Thompson, R. E., Larson, D. R. \& Webb, W. W. Precise nanometer localization analysis for individual fluorescent probes. Biophysical Journal 82, 2775-83 (2002). .

[2] Dréau, A. et al. Avoiding power broadening in optically detected magnetic resonance of single NV defects for enhanced dc magnetic field sensitivity. Physical Review B 84, 1-8 (2011). .

[3] McGuinness, L. P. et al. Quantum measurement and orientation tracking of fluorescent nanodiamonds inside living cells. Nature Nanotechnology $\mathbf{6}$, 358-63 (2011). .

[4] Schröder, T., Gädeke, F., Banholzer, M. J. \& Benson, O. Ultrabright and efficient single-photon generation based on nitrogen-vacancy centres in nanodiamonds on a solid immersion lens. New Journal of Physics 13, 055017 (2011). .

[5] Maclaurin, D., Hall, L. T., Martin, a. M. \& Hollenberg, L. C. L. Nanoscale magnetometry through quantum control of nitrogen-vacancy centres in rotationally diffusing nanodiamonds. New Journal of Physics 15, 013041 (2013). 
Estimated Parameters for Freely Rotating Nanodiamonds XLabel: Number of Emitters per site,

YLabel: Laser Intensity (uW/um^2),

Contour: Exposure time per NV (us)

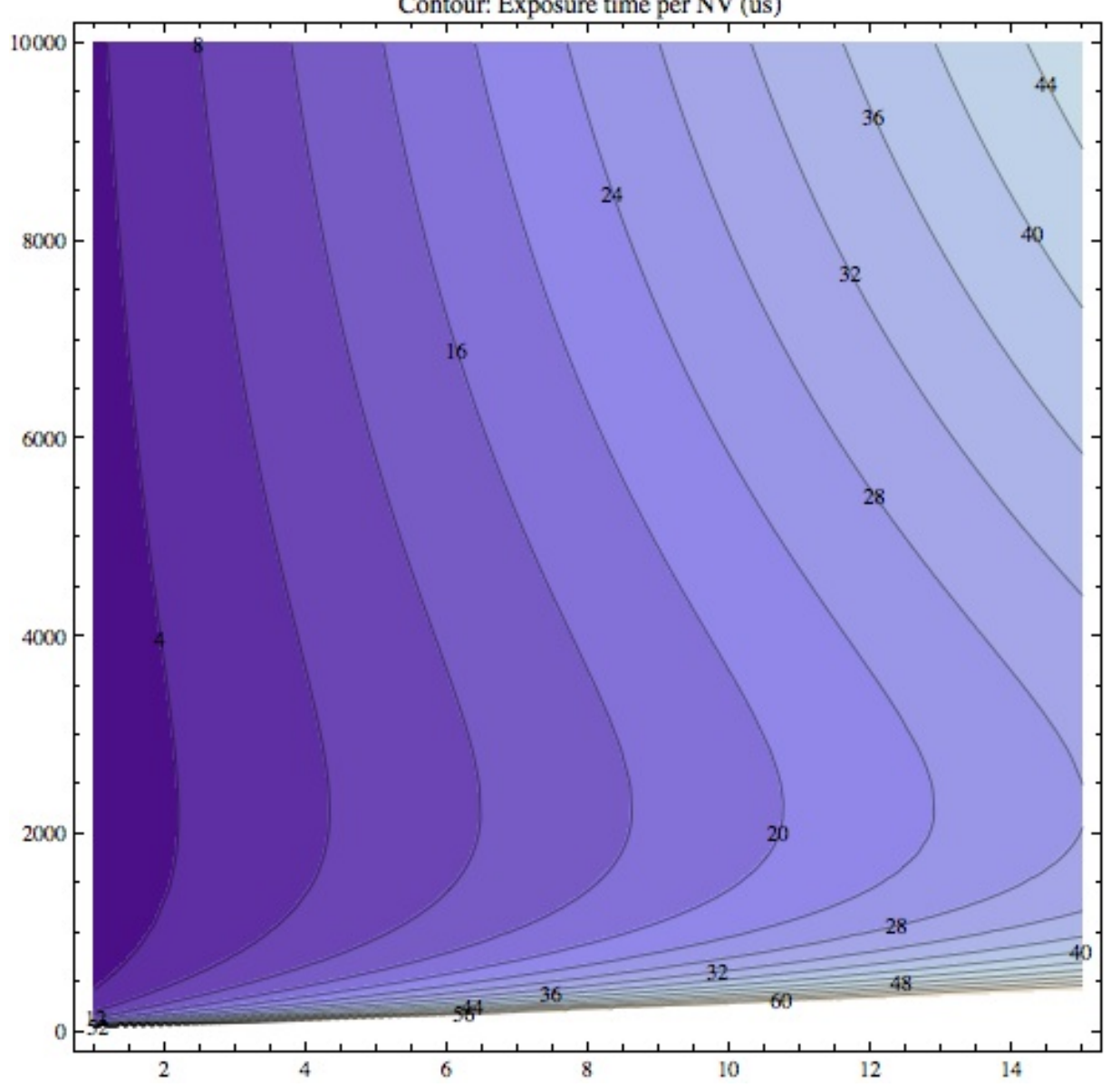

Figure S5: Estimated number of resolvable centers from freely rotating nanodiamonds. As can be seen, the more number of resolved emitters (Xaxis), the greater the exposure time (contour, $\mu$ s) needed per emitter for an ODMR signal with a unity SNR. 